\title{
Combined Emerging Capabilities for Near-Earth Objects (NEOs)
}

S.N. Milam (NASA/GSFC), H.B. Hammel (AURA), J. Bauer (UMD), M. Brozovic (JPL), T. Grav (PSI),

B.J. Holler (STScI), C. Lisse (JHU/APL), A. Mainzer (JPL), V. Reddy (Arizona), M. E. Schwamb (Gemini Obs.), T. Spahr (NEO Sciences, LLC), C.A. Thomas (NAU), D. Woods (MIT, Lincoln Labs)

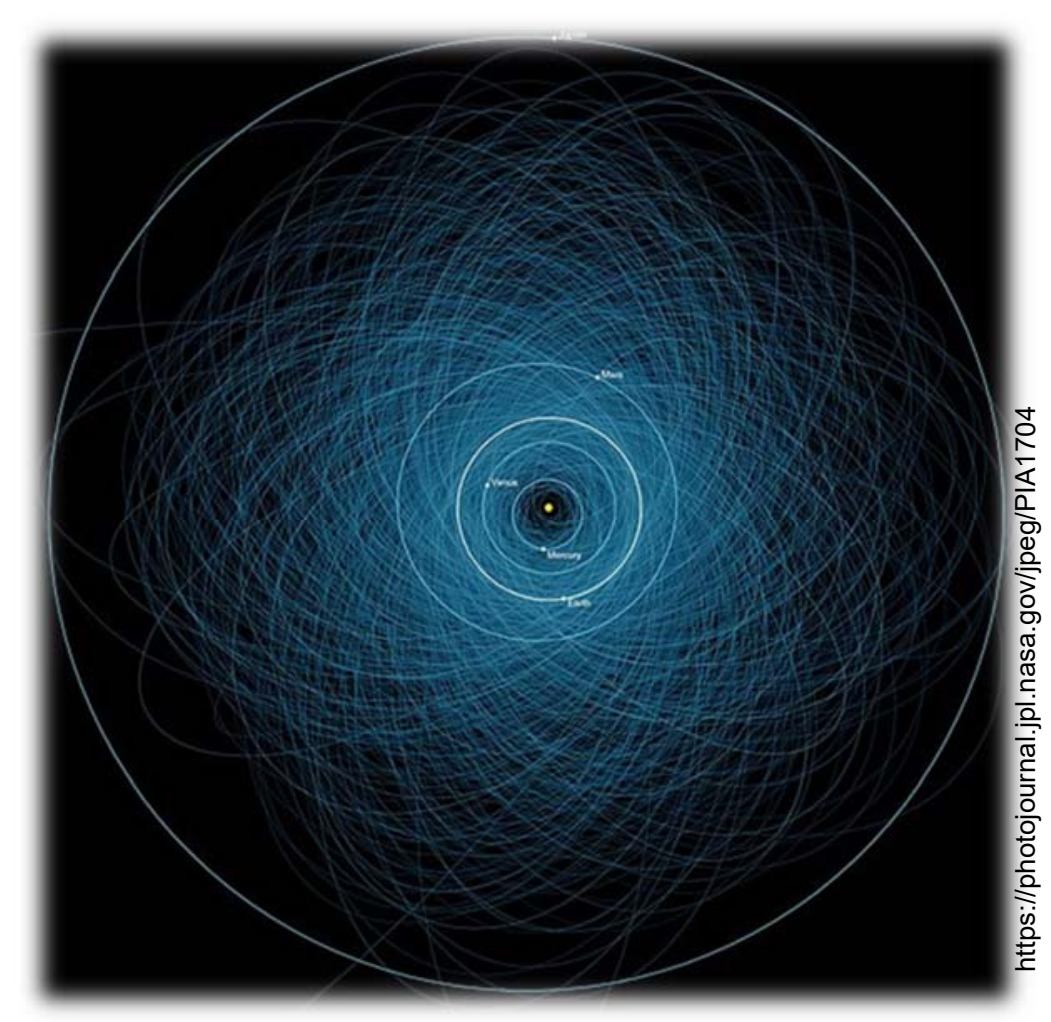

Full version of paper can be found on ArXiv:

https://arxiv.org/ftp/arxiv/papers/1907/1907.08972.pdf

Endorsements of an earlier version of this document that was submitted to Astro2020: Andrew S. Rivkin (JHU/APL), Conor A. Nixon (NASA GSFC), Aigen Li (University of Missouri), Amanda R. Hendrix (Planetary Science Institute), Glenn S. Orton (JPL), Carey M. Lisse (JHU/APL), Zhong-Yi Lin (IANCU), Michael Mueller (SRON Netherlands Institute for Space Research), Susan D. Benecchi (Planetary Science Institute), Pablo Santos-Sanz (Instituto de Astrofísica de Andalucía, CSIC), Estela Fernández-Valenzuela (FSI-UCF), Charles E. Woodward (University of Minnesota), Alvaro Alvarez-Candal (Observatório Nacional / MCTIC), James F. Bell (Arizona State University), Ronald J. Vervack Jr. (JHU/APL)Ludmilla Kolokolova (University of Maryland), Matthew S. Tiscareno (SETI Institute), Driss Takir (JETS/ARES, NASA JSC), Arielle Moullet, SOFIA/USRA, Martin Elvis (Center for Astrophysics | Harvard \& Smithsonian) 


\section{Abstract}

Near-Earth objects (NEOs) are asteroids and comets that have their perihelion distance less than 1.3 au. To date, we know about 20,000 Near-Earth Asteroids (NEAs) and just over 100 Near-Earth Comets (NEC). Potentially Hazardous Asteroids (PHAs) are NEAs that are larger than $\sim 140 \mathrm{~m}$ in diameter and that have an Earth Minimum Orbit Intersection Distance (MOID) smaller than 7.5 million $\mathrm{km}$. We currently know about 2000 PHAs. The most critical questions about PHAs are: When is the next impact likely to occur? and How bad will it be? To answer the former, we must discover PHAs in large numbers and measure their orbits with sufficient precision to understand their impact probabilities on timescales of order a century or two. The answer to the latter question depends largely on kinetic energy, which scales as $1 / 2 \times$ mass $\times$ velocity ${ }^{2}$, or as $\sim$ diameter $^{3} \times$ density $\times$ velocity ${ }^{2}$. Most survey telescopes focus on discovery of PHAs in an effort to address the first question, with little effort to provide information about the objects' impact energy beyond their absolute visible magnitude. Characterization efforts are restricted to a much smaller number of objects. For example, $\sim 10 \%$ of known NEOs have been spectrally characterized; (Binzel et al. 2019) and $\sim 10 \%$ of these NEOs are also PHAs

\section{Current NEO Capabilities}

Survey: Current dedicated NEO surveys are primarily conducted by ground-based telescopes operating at visible wavelengths with 1-2 m class apertures, with the exception of the WISE/NEOWISE mission, which operates at mid-infrared (IR) wavelengths from low-Earth orbit. Survey completeness exceeds $90 \%$ for NEAs larger than $1 \mathrm{~km}$ in effective spherical diameter and is estimated at $\sim 30 \%$ for NEAs larger than $140 \mathrm{~m}$ (Stokes et al. 2017). However, completeness is of order $1 \%$ or less for objects of the scale of the Tunguska and Chelyabinsk impactors $(\sim 20-50$ $\mathrm{m})$. Table 1 summarizes the currently operating surveys.

Another facility expected to become operational within the next year include the Space Surveillance Telescope (SST; Monet et al. 2013). Technical specifications are restricted due to its primary funding source (DARPA), but its aperture is $3.5 \mathrm{~m}$ with a $6 \mathrm{sq}$ deg FOV. The SST is currently being transported to Australia for recommissioning. Its primary mission objective is not NEO-related, requiring fast ( $\leq 2 \mathrm{sec}$ ) exposures, and negotiations for access to the NEO data with the sponsoring agency (U.S. Space Force) are underway.

Characterization: Impact energy scales as diameter ${ }^{3} \mathrm{x}$ density $\mathrm{x}$ velocity ${ }^{2}$. While velocity comes from knowledge of orbit, impact energy depends strongly on diameter and less so on density. Thus, knowledge of diameter is critically important to determining impact energy; a factor of 2 diameter uncertainty translates to nearly an order of magnitude uncertainty in impact energy. However, efforts to understand anything beyond an object's orbit and absolute visible magnitude, $\mathrm{H}$, are restricted to a much smaller sample: of the $\sim 20,000$ NEOs known today, only a few thousand have been the subject of characterization efforts. Other parameters such as rotational period, spin pole orientation, bulk density, surface properties, shape, and potential satellites will also influence mitigation strategies should an object with a high impact probability be discovered. Different observational methods yield differing parameters, summarized in Table 1. 
Effective spherical diameters are most directly determined using radar or IR radiometry and can be measured to within $\sim 10-20 \%$ with good quality data (Wright et al. 2018, Mainzer et al. 2011, Nolan et al. 2013, Tedesco et al. 2002); stellar occultations also obtain diameters, but these observations tend to be limited for NEOs (Herald et al. 2018). Direct measurements of density are known for a handful of NEOs. These are derived from in situ spacecraft visits (e.g. Itokawa; Yoshikawa et al. 2007), from radar and optical observations of the Yarkovsky effect candidates (Chesley et al., 2003; Chesley et al., 2014), and from radar observations of binary NEAs (e.g. Margot et al., 2002). To date, radar observed 57 out of 64 known binaries in the NEA population, and at least $50 \%$ of the observed objects have data suitable for density estimates. Similar to albedo and colors, density is broadly correlated with taxonomic class.

\section{Summary of Emerging Asset Capabilities:}

\subsection{NEO Searches}

While the current surveys cannot reach $90 \%$ completeness for potentially hazardous asteroids (PHAs) $\geq 140 \mathrm{~m}$ within the next few decades, emerging assets will provide some capability. It has been assumed that absolute visible magnitude $\mathrm{H}<22$ mag is an accuracy proxy for objects that are $\geq 140 \mathrm{~m}$; this assumes that all NEOs have albedos of $14 \%$. However, Wright et al. (2017) showed that $\sim 25 \%$ of NEOs have extremely low albedos, around $3 \%$, and the remaining two-thirds are in a moderately dark population, around $17 \%$. Using the size distribution of NEOs from Ivezic et al. (2002), Wright et al. (2017) demonstrated that a survey that aims to be $90 \%$ complete in $\mathrm{H}$ must reach that completeness for $\mathrm{H}<23$ mag to ensure that $90 \%$ of the objects $\geq 140$ m are detected.

Depending on its observing cadence, the predicted Vera C. Rubin Observatory (Rubin formerly LSST) completeness during its lifetime (2022-2032) is $80 \%$ for PHAs with $\mathrm{H}<22$ mag (Chesley et al. 2017, Jones et al. 2018) and 71\% for the PHAs $\geq 140 \mathrm{~m}$ (Grav et al. 2016). NearEarth Object Surveillance Mission (NEOSM - formerly NEOCam) is a dedicated NEO survey optimized for high survey completeness for PHAs $\geq 140 \mathrm{~m}$ that will also detect hundreds of thousands of smaller objects. NEOSM's expected completeness is $78 \%$ for PHAs $\geq 140 \mathrm{~m}$ during its 5 -year baseline mission, increasing to $88 \%$ if a 5 -year extended mission is included (Mainzer et al. 2015). These numbers were reproduced in the NASA Report of the Near-Earth Object Science Definition Team named "Update to Determine the Feasibility of Enhancing the Search and Characterization of NEOs" (Stokes et al. 2017). This report reaffirmed that the goal of the search system should be to produce a catalog that is $90 \%$ complete for PHAs $\geq 140 \mathrm{~m}$, and found that the most cost-effective way to achieve this goal is using a half-meter IR space-based system or a combined visible and IR system located at the L1 Sun-Earth Lagrange point.

Other current or emerging assets, such as the Transiting Exoplanet Survey Satellite (TESS), the James Webb Space Telescope (JWST), and the Nancy Grace Roman Space Telescope (Roman - formerly WFIRST), will make negligible contributions to the search effort. The JWST and Roman have very limited fields of view, which makes searching for PHAs nearly impossible for JWST and limited with Roman. While TESS has a very large field of view, its limited sensitivity and very large pixel sizes (21" per pixel) makes searching for PHAs nearly impossible. 


\subsection{NEO Characterization}

The emerging assets offer some capability to characterize PHAs. TESS monitors the same $96 \times 24 \mathrm{deg}^{2}$ field of view for 27 days, providing postage stamps of 200,000 preselected stars every 2 minutes and full frame images at 30-minute cadence. TESS will provide well-sampled rotational light curves for most of the brighter main belt asteroids and a much smaller number of PHAs, as these rarely are bright enough for TESS to detect. TESS will also provide information on thousands of stellar occultation events (Pal et al. 2018), but PHA occultations will be limited.

Rubin will provide a catalog of observations of PHAs from $\sim 0.3-1.1 \mu \mathrm{m}$ (ugriz), allowing for colors to be determined for some objects. This can allow for crude determination of taxonomic classification of many PHAs using the taxonomic system of e.g. Carvano et al. (2010). However, this effort will be made significantly harder by the cadence, which results in different filters being taken days, months, or years apart, which means that effects such as rotation and phase curves can significantly affect the resulting colors and classifications. For some objects, rotation state and phase curves may be solvable.

NEOSM will provide observations in thermal IR wavelengths for most PHAs $\geq 140 \mathrm{~m}$. IR observations allow for determination of diameter (Usui et al. 2014, Mainzer et al. 2011, Tedesco et al. 2002), a key physical parameter in the impact hazard, since impact energy scales as diameter ${ }^{3}$. While diameter can be determined using IR photometry alone, if visible photometry is also available, the combination of visible and IR measurements allows albedo to be determined. The combination of optical photometry from Rubin and thermal IR from NEOSM provides a powerful dataset that will yield accurate diameters and albedos for the majority of PHAs $\geq 140 \mathrm{~m}$. NEOSM also includes support for a targeted mode that allows high SNR observations in two channels to be obtained, along with densely sampled lightcurves.

JWST can potentially characterize surface composition, surface alteration, and physical properties of individual PHAs using visible and near-IR (NIR; 0.7-2.5 $\mu \mathrm{m}$ ) wavelengths (Thomas et al. 2016). While JWST should be able to perform sidereal tracking of a large fraction of NEOs, it is unclear how much of the observing time will be available devoted to NEOs. Additionally, observing overheads are expected to be large ( $\sim 5 \mathrm{~min})$ compared to the exposure time required for most NEOs ( few min). Thus, JWST will be of tremendous value for study of individual PHAs of particular interest, but will see limited usefulness in the characterization of more than a small number of objects.

\section{Gaps in Capabilities}

\subsection{Future Surveys}

The exact Rubin cadence and survey region are not yet determined, but there is some chance it will extend to $+10 \mathrm{deg}$ ecliptic latitude (LSST Science Collaboration, 2017). Rubin is in the southern hemisphere, so image quality will suffer at moderate northern ecliptic latitudes. High northern ecliptic latitudes will be entirely unobservable at faint $(\mathrm{V}>23)$ magnitudes. This uncovered area will reduce NEO completeness for $\geq 140 \mathrm{~m}$, and will also decrease the ability to 
warn of imminent impacts from small $(<100 \mathrm{~m})$ NEOs appearing on their last approach in the northern hemisphere. No future survey currently planned will achieve high survey completeness in this smaller size scale because there are so many objects; however, enough objects will be discovered by Rubin and NEOSM to enable robust statistics on the population's characteristics.

\subsection{Future Characterization}

Table 2 summarizes emerging assets that could be used for NEO characterization. Outside of the diameters provided by NEOSM and broad band colors from Rubin, there is currently no guarantee of any other large-scale characterization work. In the absence of observations by an IR system, all albedos and diameters are speculative. Radar observations can obtain high-quality sizes of a few dozen objects each year, as well as put size constraints to at least several dozen more. The increasing faintness of the average NEO discovery will also result in a drop in objects characterized at optical wavelengths (e.g. colors, lightcurves, and spectra), although at least some of these objects will have future observing opportunities.

Given the limitations on rate of motion and solar elongation of both JWST and Roman (see Thomas et al. 2016; Holler et al. 2018), there will always be objects that cannot be observed by either telescope. Further, time is not guaranteed on either facility for this work. NEOs frequently require immediate characterization observations, and this may not be possible given scheduling and observing constraints on both solar elongation and rate of motion.

Rotation state determination requires observations across many geometries and timescales, or radar. These observations are possible for a few hundred objects per year, extending this to a larger study of hundreds or thousands of NEOs will not be possible given the available observing time. While TESS covers large areas of sky over long ( 27 day) timeframes, its limiting sensitivity of $\mathrm{V} \sim 19$ means that few additional light curves beyond those currently available are likely to be obtained. Likewise, visible and near-IR (VNIR) spectroscopy for many thousands of objects will also be unlikely to occur given the competitive nature of observing proposals and the amount of time available. Opportunities for large amounts of time on 8-meter class telescopes may arise as the next generation of Extremely Large Telescopes come on-line in the 2020s.

NIR is essential for constraining composition, density and meteorite analogs of PHAs. While taxonomic classification is possible with broadband colors and visible spectra, it is not diagnostic of surface composition (Gaffey et al. 2002). At present, taxonomic classification using colors is limited to objects brighter than $\mathrm{V} \sim 21$ and $\mathrm{V} \sim 19.5$ for detailed surface characterization using NIR spectroscopy. NIR characterization is limited to objects whose positional uncertainties are $<1$ arcminute due to the limited field of view of the guide camera on the most prolific instrument used for this task (NASA IRTF). As Rubin and NEOSM come online, the number of targets available for characterization each night is expected to increase exponentially, making it challenging to keep up with detailed characterization of a significant fraction of small PHAs. Additional ground- and space-based assets for characterization of NEOs would help keep characterization in pace with discovery. 
A new astrophysics mission was recently selected, SPHEREx, which is 1-5 $\mu$ m all-sky survey mission that will detect $\sim 100,000$ (MBA + NEO) asteroids. SPHEREx will have the sensitivity of WISE but in 100 spectral channels. NEO full spectra will be challenging if the target is moving rapidly transverse to the ecliptic, but asteroid spectra will be easily obtained.

The Goldstone and Canberra radar antennas are primarily dedicated to spacecraft tracking, and time to observe NEOs is negotiated with spaceflight projects. Currently, Goldstone devotes about $6 \%$ of its time to NEA radar observations. Demands on the $70 \mathrm{~m}$ antenna for spacecraft tracking have been shrinking, so more time at DSS-14 is likely to become available in the future. At Arecibo, the Environmental Protection Agency (EPA) limits radar use to 1000 hours/year, and any increase beyond that would need to be renegotiated with the EPA.

\section{Summary}

Next-generation surveys can significantly increase the number of NEOs discovered and tracked, but the number of objects is expected to greatly outpace the ability of current facilities to determine their impact energies and physical properties. Survey facilities will provide sparse colors and lightcurves and, if thermal IR is available, diameters, albedos, and thermal inertias along with rotational states and lightcurves. For detailed taxonomic characterization via spectroscopy, future planned facilities such as JWST will offer some modest improvement in the number of objects available today. The availability of radar facilities is not expected to change dramatically in the coming decade.

\section{References:}

Binzel, R.P. et al. 2019, Icarus, 324, 41.

Carvano, J. M et al. 2010, A\&A, 510, 43.

Chesley, S.R. et al. 2014, Icarus, 235, 5.

Chesley, S.R. et al., 2003, Science, 302, 5651, 1739.

Gaffey M. J., et al. 2002, In Asteroids III (W. F. Bottke Jr. et al., eds.), 83. UA, Tucson.

Grav, T., et al. 2016, AJ, 151, 172.

Herald, D., et al. 2018, Asteroid Occultations V2.0. NASA Planetary Data System.

Holler, B. J et al. 2018, JATIS 4, 034003.

LSST Science Collaborations. 2017, https://github.com/LSSTScienceCollab orations/ObservingStrategy.

Magnuson, M., et al. 2018, American Astronomical Society, DPS meeting \#50, id.312.07

Mainzer, A., et al. 2010, ApJ 736, 100.

Margot, J. L et al. 2002, Science, 296, 1445.

Naidu, S.P., et al. 2016, AJ 152, 99.

Nolan, M.C., et al. 2013, Icarus 226, 629-640.
Pál, A., et al. 2018, PASP 130, 4503.

Ruprecht, J.D., et al. 2018, IEEE Aerospace Conference, 10.1109/AERO.2018.8396388.

Stokes, G., et al. 2017, Report of the Near-Earth Object Science Definition Team. Report prepared at the request of NASA.

Tedesco, E., et al. 2002, AJ 123, 1056.

Thomas, C. A., et al. 2016, PASP 128, 8002.

Usui, F., et al. 2014, PASJ 66, 56.

Warner, B., et al. 2018, Eds., Asteroid Lightcurve Data Base (LCDB) V2.0 NASA Planetary Data System.

Wright, E., et al. 2018,. arXiv:1811.01454.

Yoshikawa, M., et al. 2007, Proceedings if IAU Symposium 236. Edited by G.B. Valsecchi and D. Vokrouhlický, and A. Milani. Cambridge: Cambridge University Press, 2007., 401. 
Table 1: Characterization of NEOs is currently performed by a variety of facilities and wavelengths; however, for the vast majority of the $\sim \mathbf{1 9 , 0 0 0}$ NEOs known, little is known about them other than orbit and $\mathrm{H}$.

\begin{tabular}{|c|c|c|c|c|c|}
\hline $\begin{array}{l}\text { Method \& } \\
\text { Facilities }\end{array}$ & Parameters Determined & Aperture & $\begin{array}{l}\text { Approx. \# of } \\
\text { Objects }\end{array}$ & $\begin{array}{l}\text { Approx. } \\
\text { Sensitivity }\end{array}$ & Notes \\
\hline $\begin{array}{l}\text { Radar: Arecibo \& } \\
\text { Goldstone }\end{array}$ & $\begin{array}{l}\text { Orbit refinement, } \\
\text { diameter, shape, surface } \\
\text { features, rotational state, } \\
\text { multiplicity, radar } \\
\text { scattering properties, } \\
\text { masses for Yarkovsky } \\
\text { effect candidates, } \\
\text { constraints on optical } \\
\text { albedos and compositions; } \\
\text { for binaries: mass and } \\
\text { bulk density estimates. }\end{array}$ & $\begin{array}{l}305 \mathrm{~m} \& \\
70 \mathrm{~m}\end{array}$ & $\begin{array}{l}\text { Total unique } \\
\text { objects } \\
\text { between } \\
\text { Arecibo \& } \\
\text { Goldstone is } \\
\sim 80-110 \text { per yr }\end{array}$ & $\begin{array}{l}\text { Can get shape } \\
\text { model for } 140 \mathrm{~m} \\
\text { NEO at } @ 0.06 \mathrm{au} \\
\text { (Arecibo) \& } \\
@ 0.03 \mathrm{au} \\
\text { (Goldstone) }\end{array}$ & $\begin{array}{l}\text { Radar detectability scales as } \\
1 / \text { distance }{ }^{4} \text {. Full } \\
\text { shape/rotational state modeling } \\
\text { only possible for } \sim 30 \% \text { of } \\
\text { detected NEOs (e.g. Nolan et } \\
\text { al. } 2013 \text { ) }\end{array}$ \\
\hline $\begin{array}{l}\text { Visible \& near-IR } \\
\text { (VNIR) colors: } \\
\text { Palomar, Mayall, } \\
\text { Blanco, SOAR }\end{array}$ & Rough taxonomic class & $4-5 m$ & $\sim 500$ & $23 \mathrm{~V} \mathrm{mag}$ & $\begin{array}{l}\text { Colors can provide taxonomic } \\
\text { classification with enough } \\
\text { wavelengths \& high SNR (e.g. } \\
\text { Carvano et al. 2010). }\end{array}$ \\
\hline $\begin{array}{l}\text { VNIR } \\
\text { spectroscopy: } \\
\text { Palomar, Mayall, } \\
\text { Blanco, SOAR, } \\
\text { IRTF, Gemini }\end{array}$ & Taxonomic class & $3-8 m$ & $\begin{array}{l}\text { 2000 total } \\
\text { (including } \\
\text { IRTF) }\end{array}$ & $\begin{array}{l}21 \mathrm{~V} \text { mag }(19.5 \mathrm{~V} \\
\text { for IRTF) }\end{array}$ & $\begin{array}{l}\text { See e.g. Magnuson et al. } \\
\text { (2018). }\end{array}$ \\
\hline $\begin{array}{l}\text { Visible } \\
\text { lightcurves: } \\
\text { Modestly sized } \\
\text { telescopes }\end{array}$ & Rotational states, shapes & $<1$ to $2 \mathrm{~m}$ & $\sim 1500$ & $20 \mathrm{~V}$ mag & $\begin{array}{l}\text { Rotational state \& shape from } \\
\text { lightcurve inversion (e.g. } \\
\text { Warner et al. 2018). }\end{array}$ \\
\hline $\begin{array}{l}\text { Space- based } \\
\text { thermal IR: } \\
\text { Spitzer \& WISE/ } \\
\text { NEOWISE }\end{array}$ & $\begin{array}{l}\text { Diameters, albedos, } \\
\text { rotational states, shapes, } \\
\text { thermal inertia }\end{array}$ & $\begin{array}{l}0.4- \\
0.85 \mathrm{~m}\end{array}$ & $\sim 3000$ & $\begin{array}{l}\text { Approx. } \\
\text { equivalent to } \sim 22- \\
23 \mathrm{~V} \text { mag }\end{array}$ & $\begin{array}{l}\text { Albedo requires IR + visible; } \\
\text { thermal inertia usually requires } \\
\text { multiple viewing geometries } \\
\text { and/or shape information. }\end{array}$ \\
\hline
\end{tabular}

Note: "Diameter" refers to effective spherical diameter. Both Spitzer and NEOWISE are likely to be decommissioned in the next year. 
Table 2: Future facilities that could contribute to NEA characterization.

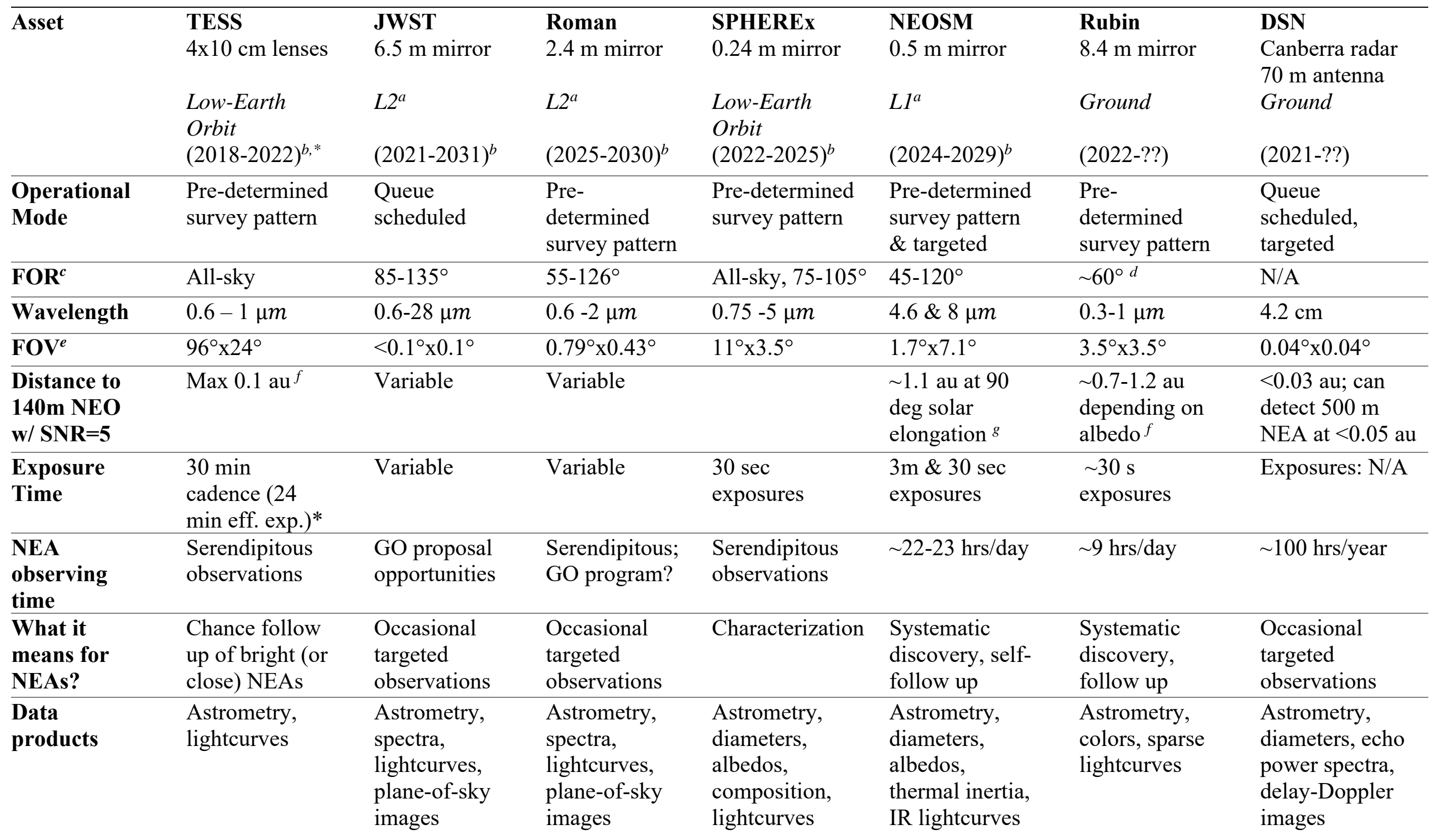

NOTE: "Follow up" means telescope will sweep NEAs discovered by other telescopes as a part of the regular operations and "self-follow up" means targeted observations of the objects that it discovered. ${ }^{a}$ Sun-Earth L1/L2. ${ }^{b}$ Primary Mission. ${ }^{c}$ Field of Regard (Solar Avoidance). ${ }^{d}$ Low elongation regions are accessible, with degradation in sensitivity. ${ }^{e}$ Field of View. ${ }^{f}$ Object at opposition. ${ }^{g}$ Little variation based on albedo (Grav et al. 2016; Mainzer et al. 2015). *Extended mission as of July 4 with full frame at 10 min (8 min eff. exp.). 